\title{
Estudio experimental del llenado de moldes pulvimetalúrgicos
}

\author{
A. Istúriz ${ }^{*, * *}$, M.D. Riera,*** y J.M. Prado,** \\ Resumen En el proceso de conformado pulvimetalúrgico, la consolidación del polvo se inicia con el \\ llenado del molde en el que se fabricará la preforma porosa, el cuál está caracterizado por la \\ distribución de densidades del polvo dentro del molde y depende tanto de las propiedades \\ del mismo como del método de llenado utilizado. A continuación, presentamos los \\ primeros resultados obtenidos mediante un montaje experimental que reproduce el llenado \\ de moldes y que, empleando un sistema de video, permite analizar el flujo de las partículas \\ durante el llenado. El objetivo es determinar el efecto del tamaño, naturaleza y morfología \\ del polvo, así como el de la velocidad del cargador y la geometría del molde en la \\ distribución de la densidad aparente en moldes industriales de geometría compleja.
}

Palabras clave Materiales granulares. Flujo granular. Distribución de densidades. Llenado de moldes.

\section{Experimental study of die filling in powder metallurgy}

\begin{abstract}
Die filling is the first step in the process of powder compaction. The density distribution depends of the powder characteristics and method of filling. In this work we present the first results obtained with different experimental methods of the filling. Results have been analyzed using a video system which permits the observation of the powder flow. The aim of the work is to determinate the effects of size, morphology and nature of the powder, as well as shoe speed, and die geometry on the apparent density distribution in industrial dies of complex geometry.
\end{abstract}

Keywords Granular materials. Granular flow. Density distribution. Die filling.

\section{INTRODUCCIÓN}

El objetivo de la pulvimetalurgia es la obtención de un componente metálico acabado a partir de la compactación y posterior sinterización de los polvos de un metal, de aquí, su amplia aplicación en diversos procesos de fabricación de componentes sinterizados $^{[1-3]}$, en los cuales, las propiedades finales del componente dependen de forma relevante de la fase de llenado de la matriz, ya que en ésta se genera una distribución de densidades no uniforme que posteriormente afecta las propiedades del compacto y de la pieza final.

La distribución de densidades dentro de la matriz depende tanto de las características del polvo metálico como del método de llenado y la forma de la matriz. La forma, morfología y granulometría del polvo son los parámetros más relevantes en el estudio de los mecanismos de flujo, debido a su in- fluencia en el grado de empaquetamiento del conjunto $^{[4]}$ y en el factor de interacción partícula-partícula. En cuanto al método de llenado, la velocidad de carga $\left(v_{\text {Shoe }}\right)$ juega el papel más importante, debido a la energía cinética y a la cantidad de movimiento impuesta al flujo, lo que repercute en la consideración de efectos inerciales. Así mismo, la forma y rugosidad de la matriz determinan la distribución de superficies friccionales con las que el material entra en contacto durante su flujo, y que definen el factor de interacción partícula-pared.

\section{LLENADO DE MOLDES CON MATERIAL GRA- NULAR}

Empleando un sistema de grabación de vídeo se estudia la descarga de polvos metálicos a diferentes velocidades de vaciado con la finalidad de comprender el mecanismo de flujo de un material granular.

(*) Universitat Politécnica de Catalunya. $^{* *}$ Centre Tecnologic de Manresa. 
Como punto de partida, se utiliza un cargador horizontal y un molde vertical, ambos rectangulares y transparentes como los mostrados en la figura 1a), para reproducir el proceso de llenado industrial de moldes pulvimetalúrgicos. Se disponen capas horizontales (de igual espesor) de arena coloreada (70 $\%<1000 \mu \mathrm{m}, 100 \mu \mathrm{m}<30 \%<500 \mu \mathrm{m}$ ) y se efectúa el llenado del molde controlando la velocidad de desplazamiento horizontal del cargador $\left(\mathrm{v}_{\text {Shoe }}\right)$.

El video capturado durante el llenado, mediante técnicas de análisis de imagen, permite realizar mediciones de los parámetros de interés, tales como el ángulo de inicio de cizalladura $\left(\theta_{\mathrm{s}}\right)$ y el ángulo principal de llenado $(\beta)$, lo que permite diferenciar entre la zona de esfuerzos de cizalladura en equilibrio y la zona en régimen de flujo, como se observa en la figura $1 \mathrm{~b}$ ).

Controlando la velocidad del cargador se realizan ensayos a diferentes velocidades para diferentes materiales granulares a fin de evaluar su comportamiento. Como se muestra en la figura 2, ensayo realizado utilizando arena coloreada a una $\mathrm{v}_{\text {Shoe }}=$ $50 \times 10^{-3} \mathrm{~ms}^{-1}$, durante el llenado de un molde, los eventos que determinan la distribución final de la densidad dentro del mismo se ven afectados, de forma directa, por el proceso de descarga que ocurre dentro del cargador.

Una pila de material granular en reposo se caracteriza por formar un ángulo $\theta$ diferente de cero respecto del plano horizontal. En una pila de material granular se pueden definir dos ángulos: el ángulo de reposo, $\theta_{\mathrm{r}}$, por debajo del cual no hay flujo, y unos grados mayor, el ángulo máximo de estabilidad, $\theta_{\mathrm{m}}$, referido por primera vez por Bagnold ${ }^{[5]}$. $\theta_{\mathrm{m}}$, también llamado ángulo de movimiento, es aquel al cual ocurren avalanchas espontáneamente en la superficie de una pila de granos, después de las cuales, el ángulo de la pendiente se relaja hasta volver al ángulo de reposo, $\theta_{\mathrm{r}}$.

Entre $\theta_{\mathrm{r}}$ y $\theta_{\mathrm{m}}$ se define una zona de biestabilidad, donde los granos que pertenecen a ella pueden tanto permanecer estáticos como fluir. La presencia de paredes laterales incrementa la estabilidad de una pila de material granular, teniendo que, para un material granular confinado entre dos paredes, $\theta_{\mathrm{r}}$ y $\theta_{\mathrm{m}}$ aumentan según disminuye la distancia entre éstas ${ }^{[6]}$.

En el caso dinámico se debe considerar el efecto de la velocidad del conjunto sobre su estabilidad y esto conlleva a definir variables dinámicas como el ángulo de inicio de cizalladura $\theta_{\mathrm{s}}$, siendo $\theta_{\mathrm{r}} \leq \theta_{\mathrm{s}} \leq \theta_{\mathrm{m}}$, como el ángulo crítico donde se inicia el flujo del material granular hacia el interior del molde (inicio de la deformación de las capas).

Así mismo, al analizar un proceso como el planteado en el modelo (con $v_{\text {Shoe }}$ relativamente baja), se observa que para los primeros instantes del llenado, el flujo másico de material granular que

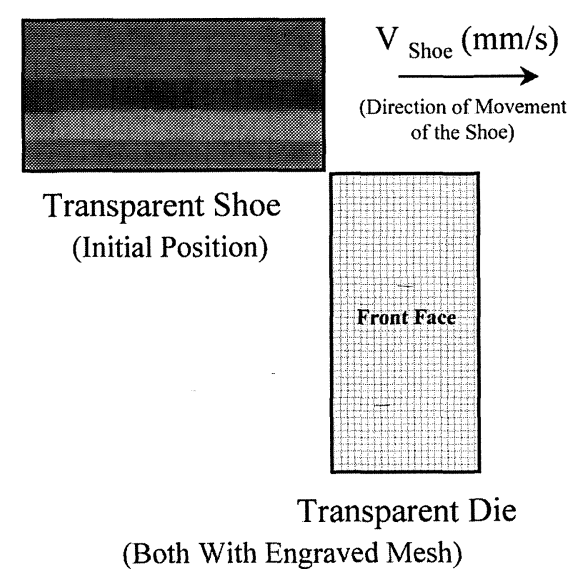

a)

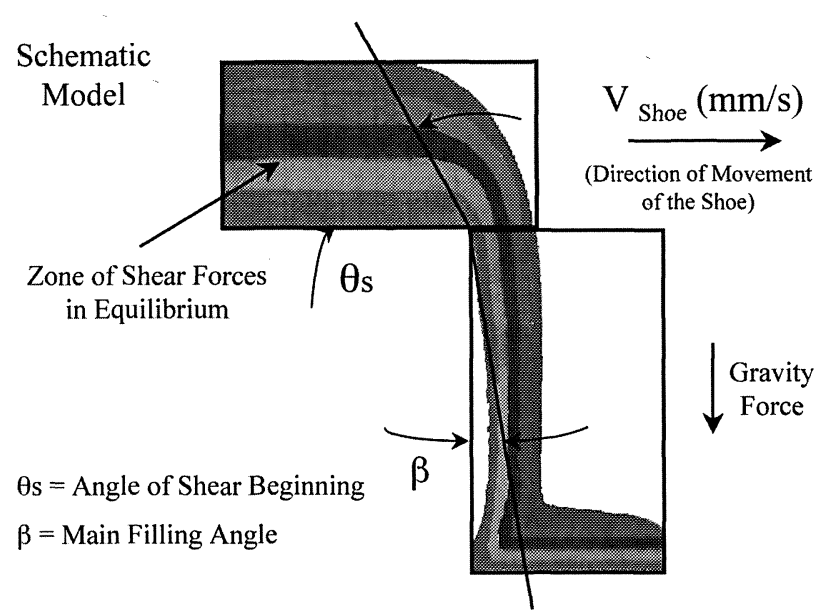

b)

Figura 1. Modelo del llenado de moldes. a) Se esquematizan las capas horizontales de arena dentro del cargador antes del llenado y la dirección de movimiento del cargador (donde $v$ shoe, es la velocidad del cargador). b) Análisis cualitativo durante el llenado: zona de esfuerzos de corte en equilibrio (donde, $\theta_{\mathrm{s}}$ es el ángulo de inicio de cizalladura y $\beta$, el ángulo principal de llenado).

Figure 1. Schematic model of die filling. a) Before de filling: horizontal layers of coloured sand inside of die and direction of movement of the shoe (where $v_{\text {Shoe, }}$ is the shoe speed). b) Qualitative analysis during the filling: zone of shear forces in equilibrium (where, $\theta_{s}$ is the angle of shear beginning and $\beta$, the main filling. 


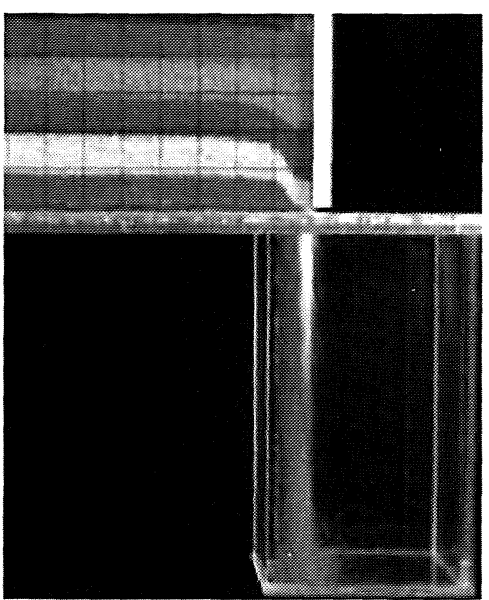

a) $\mathrm{t}=0.2083 \mathrm{~s}$

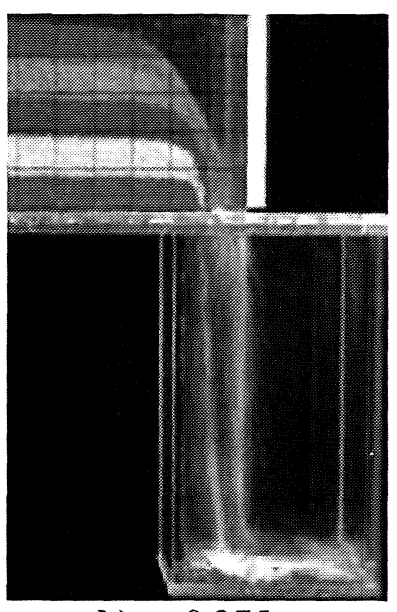

b) $t=0.375 \mathrm{~s}$

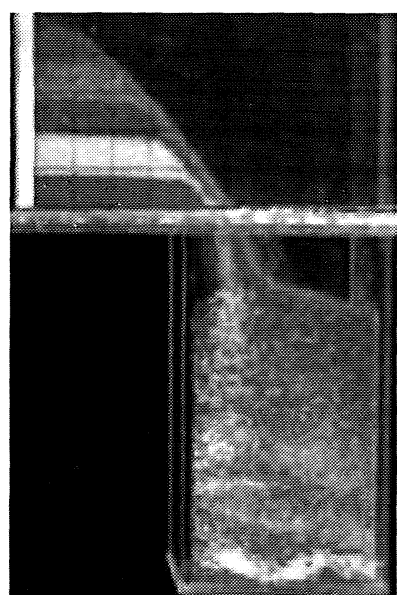

c) $\mathrm{t}=1.333 \mathrm{~s}$

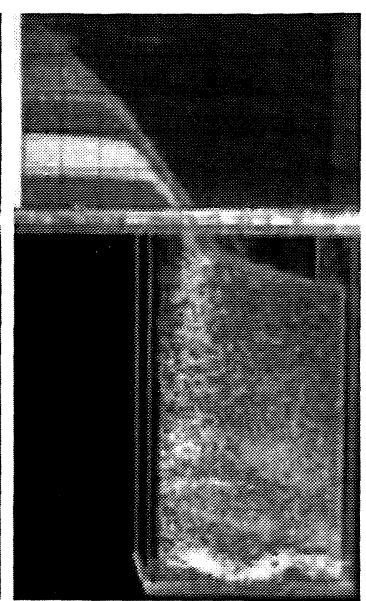

d) $\mathrm{t}=1.416 \mathrm{~s}$

Figura 2. Llenado realizado a $v_{\text {Shoe }}=50 \times 10^{-3} \mathrm{~m} / \mathrm{s}$ con arena coloreada irregular (capas horizontales), $70 \%<1.000 \mu \mathrm{m}$, $100 \mu \mathrm{m}<30 \%<500 \mu \mathrm{m}$.

Figure 2. Filling of the die with irregular coloured sand (horizontal layers), $70 \%<1,000 \mu \mathrm{m}, 100 \mu \mathrm{m}<30 \%<500 \mu \mathrm{m}$, shoe speed $50 \times 10^{-3} \mathrm{~m} / \mathrm{s}$ angle).

entra al molde es creciente, lo cual se corresponde con el incremento en el área de acceso al mismo. Seguidamente, se precipita abruptamente la cantidad de material granular que se encuentra por encima del ángulo de inicio de cizalladura, $\theta_{\mathrm{s}}$ (ver Fig. 2b)), lo que implica un máximo en el flujo másico de material que entra al molde y que, posteriormente disminuye, tendiendo a un valor estable (estacionario), asociado a las variaciones de $\theta_{\mathrm{s}}$ con el tiempo t.

Se puede observar en la figura $3\left(v_{\text {Shoe }}\right.$ ligeramente superior a la de la figura 2) que un incremento en la velocidad del cargador ocasiona un flujo másico mayor dentro del molde, y que como se aprecia en las figuras 3c) y $3 d$ ), el flujo másico estable (después de la precipitación abrupta de las figuras 3a) y 3b)) es mayor al correspondiente a la figura 2, debido al incremento másico de material por unidad de tiempo.

La distribución final de las capas de arena dentro del molde (inicialmente en forma de capas horizontales) y su evolución como función del aumento en la velocidad de flujo (proporcional a $\mathrm{v}_{\text {Shoe }}$ ) permiten distinguir fenómenos difusivos de mezcla y segregación acentuados notablemente para bajas velocidades de llenado (Fig. 4a)). Si el estado inicial corresponde a frentes de avance (Fig. 5), el flujo de material permite analizar el perfil de velocidades por encima de $\theta_{s}$, donde la velocidad de los granos se incrementa con su proximidad a la superficie libre de la zona de flujo, por efecto de la activación de la componente tangen- cial (de cizalladura) impuesta por la fuerza de la gravedad (g).

Si las geometrías del molde y el cargador son simétricas respecto a un plano vertical alineado con la dirección de carga, se puede asumir que la distribución final de los granos, y por ende la distribución de densidades dentro del molde, es simétrica respecto a dicho plano (Figs. 6 y 7). El grado de simetría de la distribución de densidades dentro del molde dependerá, entonces, del grado alineación de los elementos del sistema entre sí y respecto a la vertical, asumiendo que la densidad aparente dentro del cargador antes del llenado es totalmente homogénea.

\section{ESTUDIO DE LA DESCARGA DE POLVOS ME- TÁLICOS}

Así mismo, se pueden diferenciar dos regímenes de flujo observados durante el vaciado del cargador, un régimen de avalanchas discretas ( $\mathrm{v}_{\text {Shoe }}$ muy bajas) y un régimen continuo, observados previamente en experimentos con tambores rotatorios ${ }^{[7]}$. No obstante, la forma, morfología y tamaño de las partículas determinan, para cada material granular (seco), el tipo de régimen en función de la velocidad, conduciendo, en el caso de partículas de formas irregulares o rugosas (o en presencia de fuerzas cohesivas), a un régimen de flujo intermitente (Figs. 8 y 9), del estudio de éstos régimenes se puede derivar una velocidad crítica de llenado que garantice un llenado uniforme del molde. 


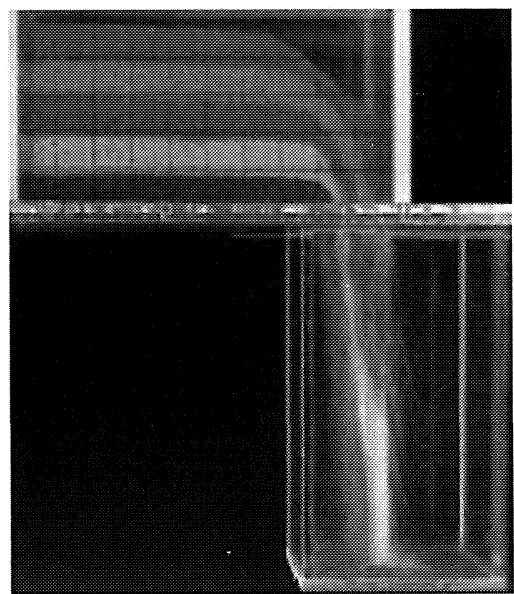

a) $t=0.2083 \mathrm{~s}$

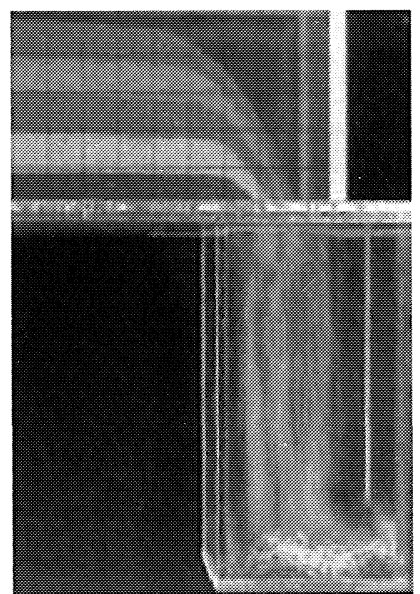

b) $t=0.3333 \mathrm{~s}$

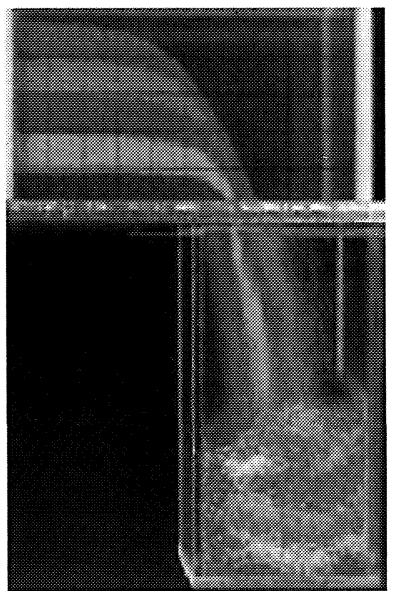

c) $\mathrm{t}=0.4583$

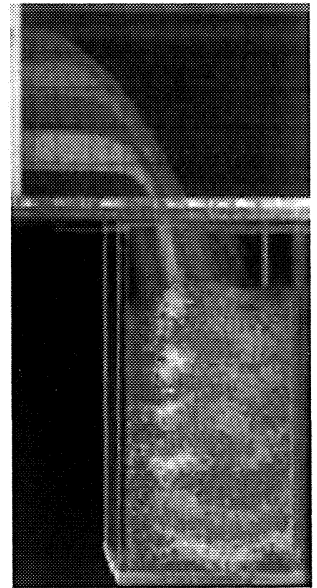

d) $\mathrm{t}=0.7083 \mathrm{~s}$

Figura 3. Llenado realizado a $v_{S h o e}=0,1 \mathrm{~m} / \mathrm{s}$ con arena coloreada irregular (capas horizontales), $70 \%<1.000 \mu \mathrm{m}$, $100 \mu \mathrm{m}<30 \%<500 \mu \mathrm{m}$.

Figure 3. Filling of the die with irregular coloured sand (horizontal layers), $70 \%<1,000 \mu \mathrm{m}, 100 \mu \mathrm{m}<30 \%<500 \mu \mathrm{m}$, shoe speed $0.1 \mathrm{~m} / \mathrm{s}$, for differ.

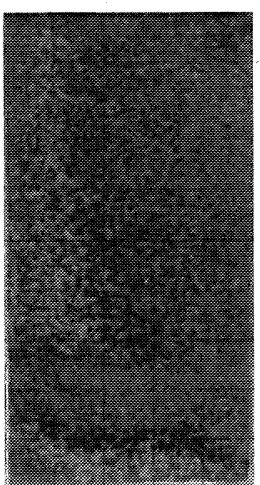

a)

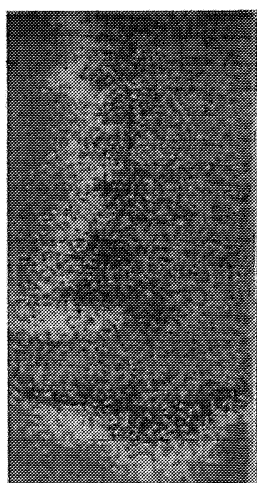

b)

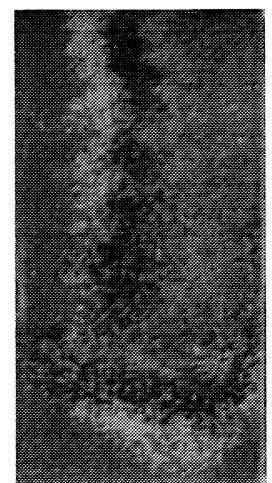

c)

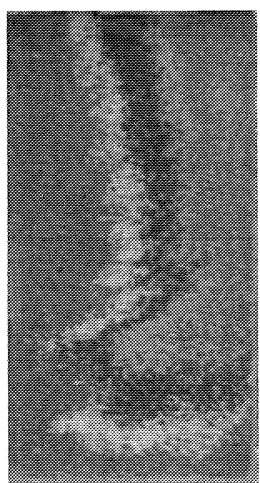

d)

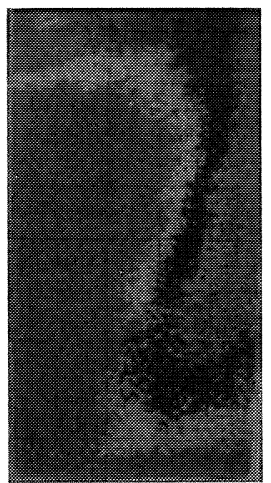

e)

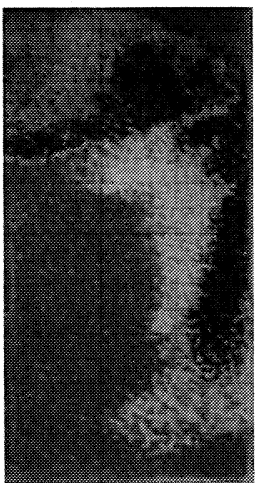

f)

Figura 4. Distribución final de las capas de arena dentro del molde después del llenado (cara frontal) para diferentes velocidades del cargador.

Figure 4. Final distribution of the coloured sand layers within the die (after filling) for different shoe speed (front face). a) vShoe= 40 $\times 10^{-3} \mathrm{~m} / \mathrm{s}$ b) vShoe $=95 \times 10^{-3} \mathrm{~m} / \mathrm{s} \mathrm{c}$ ) $v_{\text {Shoe }}=155 \times 10^{-3} \mathrm{~m} / \mathrm{s} \mathrm{d}$ ) $v_{\text {Shoe }}=245 \times 10^{-3} \mathrm{~m} / \mathrm{s}$ e) $\left.v_{\text {Shoe }}=345 \times 10^{-3} \mathrm{~m} / \mathrm{s} \mathrm{f}\right) v_{\text {Shoe }}=470 \times 10^{-3} \mathrm{~m} / \mathrm{s}$.

La humedad, al introducir fuerzas cohesivas de tipo van der Waals entre las partículas, incrementa la estabilidad del conjunto, notablemente ${ }^{[8]}$, lo que se ve reflejado en una perdida de la movilidad o la fluidez del polvo metálico. Esta, puede influir de manera apreciable en el tipo de régimen de flujo, al favorecer valores mas elevados del ángulo máximo de estabilidad, $\theta_{\mathrm{m}}$, y por consiguiente de $\theta_{\mathrm{s}}$, aumentando así el potencial disponible para un desprendimiento $\left(\theta_{\mathrm{m}}-\theta_{\mathrm{r}}\right)$, o amplitud de la avalancha, lo que conduce a un régimen de flujo intermitente.

Es de esperar que dicho potencial $\left(\theta_{\mathrm{m}}-\theta_{\mathrm{r}}\right)$, también se incremente con la disminución de la distan- cia entre las paredes del cargador, según resultados reportados previamente ${ }^{[9}$ y 10$]$. El incremento en la estabilidad del material, debido a la presencia de paredes laterales, puede ser explicado mediante la formación de arcos de partículas que direccionan una parte del peso hacia las paredes, induciendo fricción. Según el efecto Janssen, en el fondo de un recipiente, la presión en el material alcanza un punto de saturación (se asume que ésta es función de la

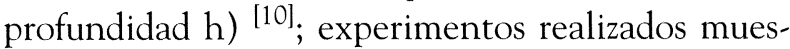
tran que la presión de saturación en un sistema bidimensional crece proporcionalmente a $\mathrm{N}^{2}$, siendo $\mathrm{N}$ la longitud de la base del recipiente ${ }^{[11]}$. 


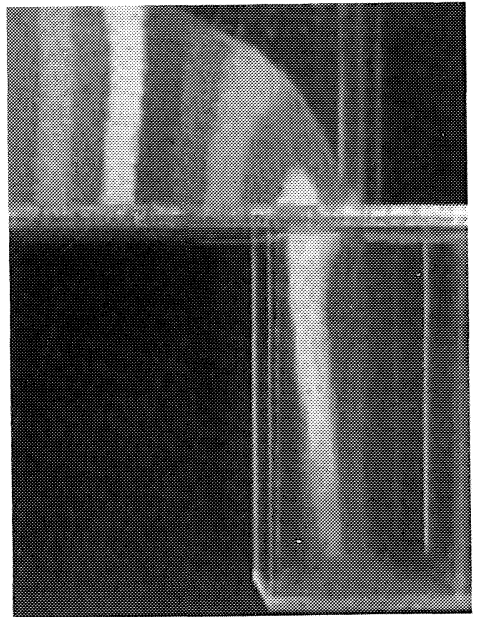

a) $\mathrm{t}=0.29 \mathrm{~s}$

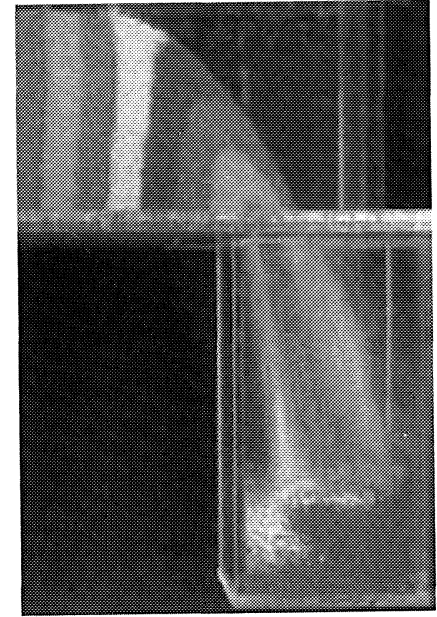

b) $\mathrm{t}=0.41 \mathrm{~s}$

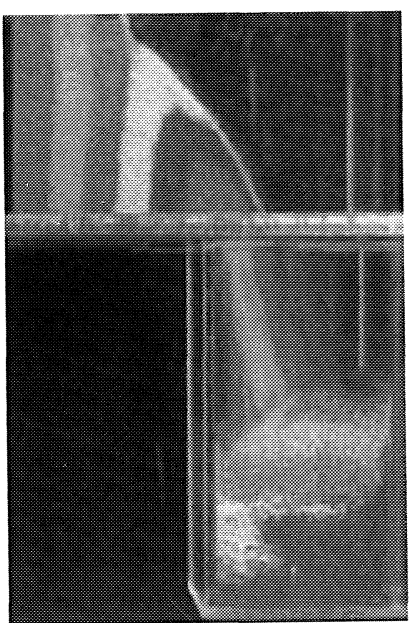

c) $\mathrm{t}=0.54 \mathrm{~s}$

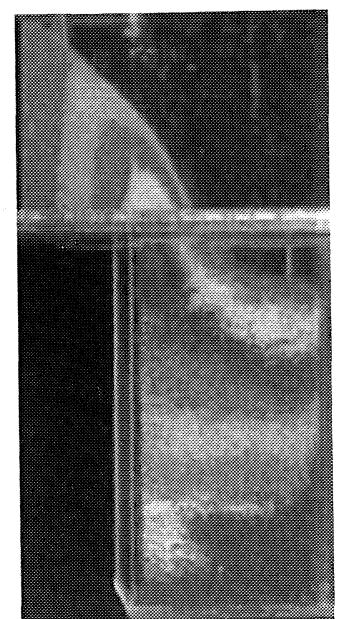

d) $\mathrm{t}=0.91 \mathrm{~s}$

Figura 5. Llenado realizado a $v_{\text {Shoe }}=0,1 \mathrm{~m} / \mathrm{s}$ con arena coloreada irregular (frentes verticales), $70 \%<1.000 \mu \mathrm{m}$, $100 \mu \mathrm{m}<30 \%<500 \mu \mathrm{m}$.

Figure 5. Filling of the die with irregular coloured sand (vertical fronts), $70 \%<1,000 \mu \mathrm{m}, 100 \mu \mathrm{m}<30 \%<500 \mu \mathrm{m}$, shoe speed $0.1 \mathrm{~m} / \mathrm{s}$, for differents times.

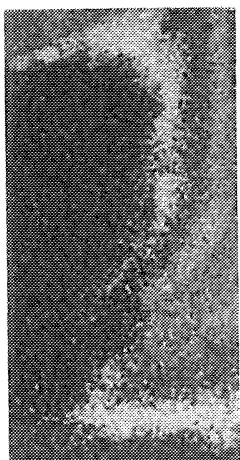

a)

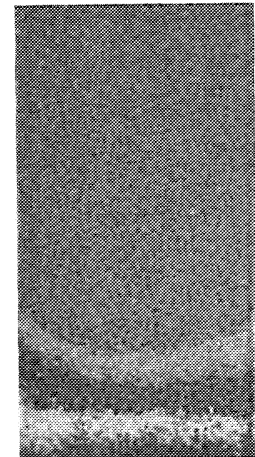

b)

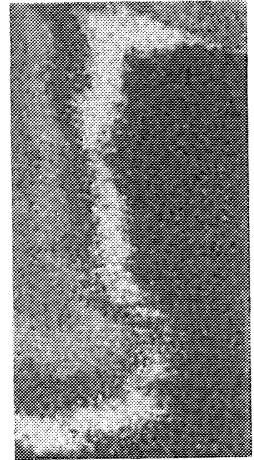

c)

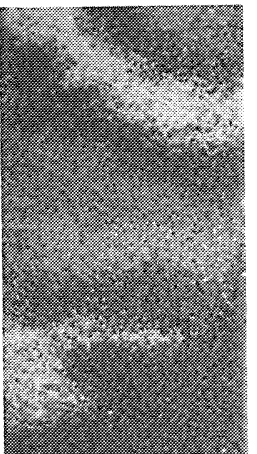

a)

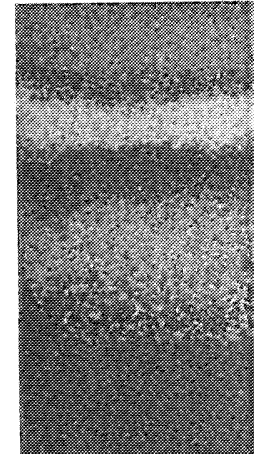

b)

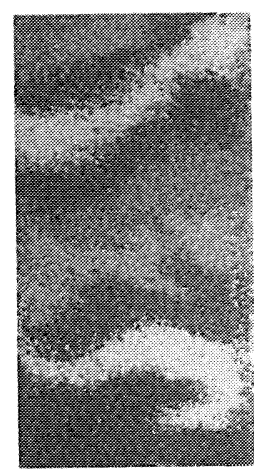

c)

Figura 6. Llenado realizado a $v_{\text {Shoe }}=0,3 \mathrm{~m} / \mathrm{s}$ con arena coloreada irregular (capas horizontales), $70 \%<1000 \mu \mathrm{m}$, $100 \mu \mathrm{m}<30 \%<500 \mu \mathrm{m}$. Vistas a) anterior b) lateral derecha y c) posterior del molde después del llenado.

Figure 6. Symmetry in the filling of the die with irregular coloured sand (horizontal layers), $70 \%<1,000 \mu \mathrm{m}$, $100 \mu \mathrm{m}<30 \%<500 \mu \mathrm{m}$, shoe speed $0.3 \mathrm{~m} / \mathrm{s}$. Views of the die a) front face b) right lateral face c) back face.

\section{CONCLUSIONES}

La distribución de densidades dentro de un molde pulvimetalúrgico previo a la compactación está afectada de manera directa, tanto por las propiedades del polvo como por el sistema de llenado y la forma del molde; sin embargo, analizando los régimes de flujo durante el llenado es posible obtener una velocidad crítica de llenado $\left(\mathrm{v}_{\text {Shoe }}\right)$ en función

de las propiedades características del polvo, que garantice un llenado uniforme del molde, considerando así mismo, las relaciones dimensionales entre el molde y el cargador, la distribución de las superficies friccionales dentro del molde y el efecto inconveniente de la humendad al modificar la fluidez de un polvo seco o lubricado.

Figura 7. Llenado realizado a $v_{\text {Shoe }}=0,1 \mathrm{~m} / \mathrm{s}$ con arena cooreada irregular (frentes verticales), $70 \%<1.000 \mu \mathrm{m}$ $100 \mu \mathrm{m}<30 \%<500 \mu \mathrm{m}$. Vistas a) anterior b) lateral derecha y c) posterior del molde después del llenado.

Figure 7. Symmetry in the filling of the die with irregular coloured sand (vertical fronts), $70 \%<1,000 \mu \mathrm{m}, 100 \mu \mathrm{m}<$ $30 \%<500 \mu \mathrm{m}$, shoe speed $0.1 \mathrm{~m} / \mathrm{s}$. Views of the die a) front face b) right lateral face and c) bac. 


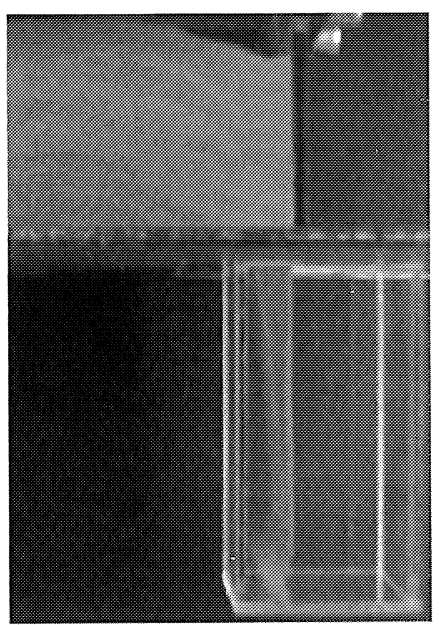

a) $\mathrm{t}=0.125 \mathrm{~s}$

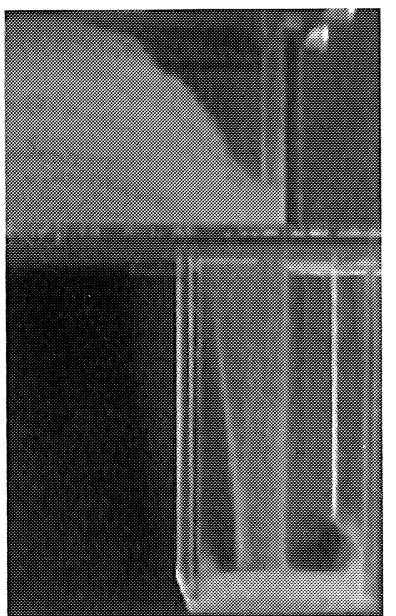

b) $t=0.25 \mathrm{~s}$

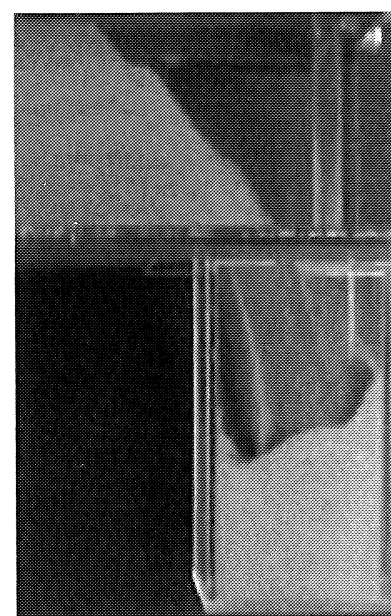

c) $\mathrm{t}=1.04 \mathrm{~s}$

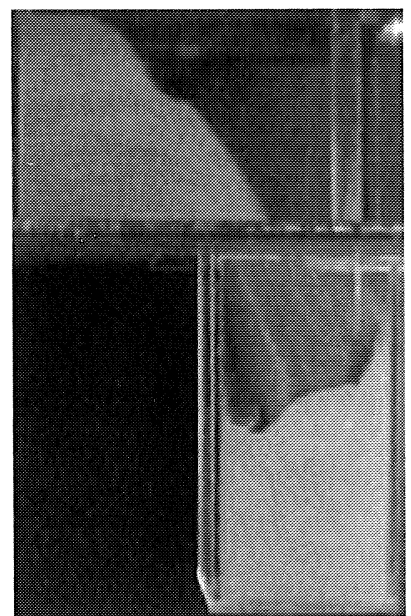

d) $\mathrm{t}=1.08 \mathrm{~s}$

Figura 8. Llenado realizado a vs $=50 \times 10^{-3} \mathrm{~m} / \mathrm{s}$ con polvo de cobre esferoidal, $100 \%<315 \mu \mathrm{m}$.

Figure 8. Filling of the die with spheroidal copper powders $(100 \%<315 \mu \mathrm{m})$, shoe speed $50 \times 10^{-3} \mathrm{~m} / \mathrm{s}$.

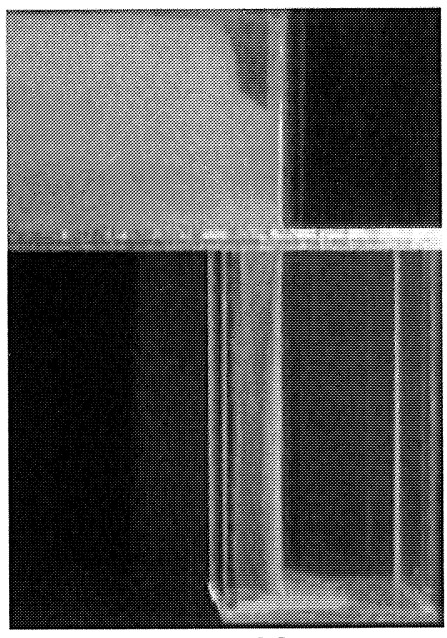

a) $\mathrm{t}=0.28 \mathrm{~s}$

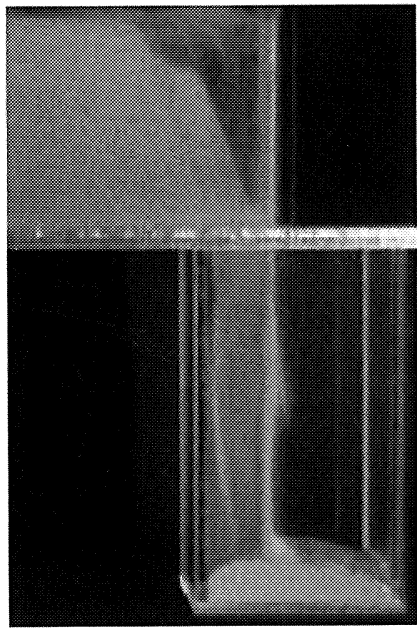

b) $\mathrm{t}=0.44 \mathrm{~s}$

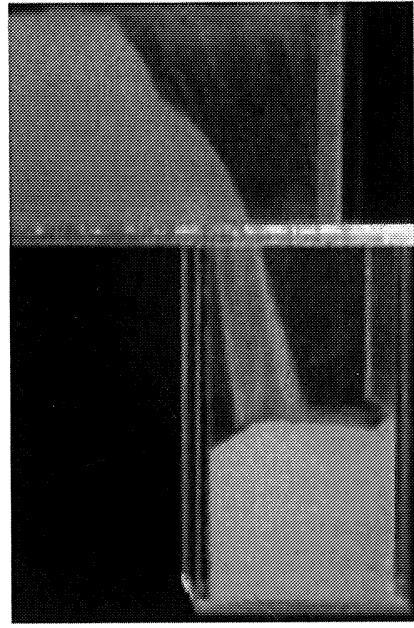

c) $\mathrm{t}=0.80 \mathrm{~s}$

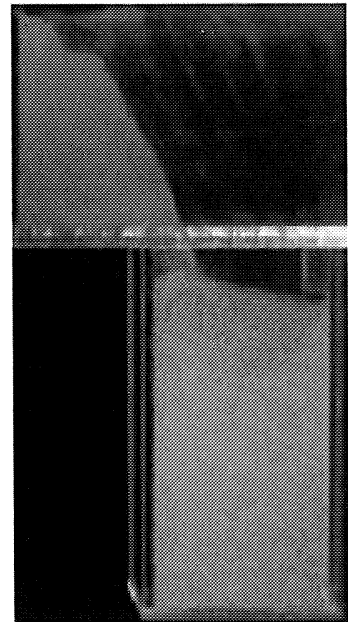

d) $\mathrm{t}=0.84 \mathrm{~s}$

Figura 9. Llenado realizado a vs $=50 \times 10^{-3} \mathrm{~m} / \mathrm{s}$ con polvo de hierro irregular; $85 \%<50 \mu \mathrm{m}, 15 \%<212 \mu \mathrm{m}$.

Figure 9. Filling of die with irregular iron powders $(85 \%<50 \mu \mathrm{m}, 15 \%<212 \mu \mathrm{m})$, shoe speed $50 \times 10^{-3} \mathrm{~m} / \mathrm{s}$.

\section{Agradecimientos}

Este proyecto está subvencionado por el MCYT (Referencia: DPI2001-2256-C02-01)

\section{REFERENCIAS}

[1] B. Torres, A. García-Escorial, J. IbáÑez y M. Lieblich, Rev. Metal. Madrid 37 (2001) 225-229.

[2] E. Gordo, M.A. Martínez, J.M. Torralba y J.A. JimÉnEZ, Rev. Metal. Madrid 37 (2001) 119-123.

[3] U.D. Hunicke, P. Adeva, W. Kowalski, P. Acosta, G. Schulz y K.H. Mundt, Rev. Metal. Madrid 37 (2001) 93-97.
[4] A. Van-Burkalow, Bull. Geol. Soc. Am., 56 (1945) 669 674.

[5] R.A. Bagnold, Proc. R. Soc. London, Ser. A, 225 (1954) 49-52.

[6] C. Liu, H.M. Jaeger y S.R. Nagel, Phys. Rev. A, 43 (1991) 7.092-7.096.

[7] J. Rajchenbach, Phys. Rev. Lett., 65 (1990) 2.221-2.225.

[8] L. BocQuet et al., Nature, 396 (1998) 735-738.

[9] P. EvesQue, Phys. Rev. A, 43 (1991) 2.720-2.723.

[10] S. Courrech du Pont, P. Gondret, B. Perrin y M. RABAUD, Europhys. Lett., 61 (2003) 494-495.

[11] R. Peralta-Fabi, C. Málaga y R. Rechtman, Europhys. Lett., 45 (1999) 76-82. 